\title{
Teoría de sistemas sociales e historia: un acercamiento interdisciplinario para la investigación científica
}

\section{Social Systems Theory and History: An Interdisciplinary Approach for Scientific Research}

\author{
Mónica Eugenia Moreno Rubio \\ Universidad Autónoma de Querétaro \\ monica.moreno@uaq.mx
}

DOI: 10.24901/rehs.v40i159.425

\section{(cc) EY-NC}

Teoría de sistemas sociales e historia: un acercamiento interdisciplinario para la investigación científica por Mónica Eugenia Moreno Rubio se distribuye bajo una Licencia Creative Commons Atribución-NoComercial 4.o Internacional.

Fecha de recepción: 24 de abril de 2018

Fecha de aprobación: I2 de noviembre de 2018

\section{RESUMEN:}

La teoría de los sistemas sociales (TSS) tenía pocos años de ser utilizada en sociología cuando recibió diversas críticas. En los años ochenta del siglo pasado, el sociólogo español Francisco Parra Luna respondió a las más importantes como aquella que sostenía que la teoría de sistemas era ahistórica. El objetivo de este artículo consiste en actualizar la contraargumentación a dicha crítica ofreciendo cuatro elementos clave que consideramos la resuelven: el modelo procesal, la teoría de los sistemas axiológicos, la dialéctica y los mecanismos sociales. En las cuatro propuestas sostenemos que la teoría de sistemas y la historia son compatibles en diversos aspectos y se necesitan entre sí en otros. La metodología utilizada consistió en la revisión documental teórica con el propósito de encontrar los elementos en los que convergen la teoría de sistemas y la historia.

Palabras clave:

Teoría de sistemas, historia, modelo procesal, sistemas axiológicos, dialéctica. 


\section{ABSTRACT:}

Social systems theory (TSS) had been applied in sociology for only a few years when it received several critiques. In the 1980 , the Spanish sociologist Francisco Parra Luna responded to the most important questionings, including one which argued that systems theory is ahistorical. The purpose of this article is to update the reply to that critique by providing four key elements that, in our view, resolve the issue: a processual model, axiological systems theory, dialectics, and social mechanisms. Based on these four proposals, we sustain that systems theory and history are compatible in many cases, and actually need one another in others. The methodology consisted in reviewing theoretical documents in order to identify those elements where systems theory and history converge.

\section{Keywords:}

Systems theory, history, processual model, axiological systems, dialectics.

\section{Introducción}

En un artículo publicado hace más de treinta años por Francisco Parra Luna en el que discute la pertinencia del uso de la teoría de sistemas en sociología_considerándola el "enfoque sociológico por excelencia"-, el autor hace un breve recuento de las críticas que ha enfrentado este enfoque. Una de ellas se refiere a su carácter ahistórico, donde el propio autor sostiene que dicha crítica se debe a que se ha confundido a la tradición teórica estructuralista con la sistémica.

Actualmente, la crítica sobre la ahistoricidad del enfoque sistémico persiste. Uno de los teóricos más renombrados, referencia obligada en teoría de los sistemas sociales, es Niklas Luhmann. Es precisamente en sus teorías donde este cuestionamiento sigue sosteniéndose,_además de otros que versan sobre la ausencia de sujetos en su modelo teórico,_ ${ }_{2}$ incluyendo el debate que Habermas sostuvo en este mismo sentido, a lo cual se agrega que aun existe incertidumbre sobre las dinámicas de interacción humana a nivel micro dentro de los sistemas. ${ }^{4}$

Por ello, no será sobre el enfoque sistémico de Luhmann que desarrollaremos nuestras propuestas debido a su elevada abstracción que complica comunicarla con una disciplina tan concreta y humana como la historia, ${ }_{-}^{5}$ sino que acudiremos a los fundamentos teóricos del enfoque sistémico y retomaremos aquellas aportaciones más recientes que, por su flexibilidad en los diversos niveles de análisis, nos ofrecen un panorama más amable para la inclusión de la historia. Consideramos que la propuesta inicial de Walter Buckley nos ofrece ciertos elementos para lograrlo dentro de su modelo procesal, así como las más recientes aportaciones de Francisco Parra Luna_en su teoría de los sistemas axiológicos y en la investigación realizada sobre El lugar de la Mancha; además, tenemos la discusión de Pablo González Casanova y adicionalmente otros teóricos analíticos que abordan los mecanismos sociales. 
Proponemos como hipótesis que es insuficiente distinguir al estructural funcionalismo de la TSS para salvar el obstáculo de la ahistoricidad; es fundamental plantear de qué manera podría insertarse la historia en los sistemas complejos y consideramos que un primer paso es abrir los canales de diálogo interdisciplinario e interteórico entre la teoría de los sistemas sociales y la historia desde distintos niveles de análisis.

Para fundamentar nuestro argumento, haremos una breve discusión que se dividirá en cuatro secciones: la primera versará sobre el modelo procesal, en la que recurriremos a una breve descripción de los modelos estructuralistas señalando en dónde se considera a la historia, pero se le otorga un papel limitado, en especial, respecto de Durkheim y Parsons. Explicaremos las bondades del modelo procesal que centra su atención en procesos; posteriormente argumentaremos en qué parte del mismo modelo se inserta la historia como elemento necesario para lograr una comprensión y explicación más completa de un fenómeno.

La segunda sección abordará la propuesta del modelo de sistemas axiológicos elaborado por Francisco Parra Luna, modelo en cuyos inputs la historia tiene un papel importante por desempeñar al ser incluida como "nivel de memoria" en el conjunto de condicionantes del sistema de valores. Posteriormente, abordaremos la discusión sobre la posibilidad de la comunicación entre el pensamiento dialéctico y la TSS, donde Pablo González Casanova es un importante promotor en este sentido; igualmente, analizaremos el carácter intrínsecamente histórico de este tipo de pensamiento que sería adecuado para aplicarse junto con el sistémico.

Para finalizar, cerraremos analizando el papel de la historia en los modelos de mecanismos sociales que pueden utilizarse en este enfoque teórico, citando como ejemplos aquellos propuestos por Donald Davidson, Peter Hedström y Jon Elster, quienes si bien no hacen referencia explícita a la historia, consideramos que al haberse volcado esta disciplina también hacia los actores individuales y sus acciones, consideramos que existen importantes determinantes históricas para explicar quién es un actor y por qué actúa como lo hace.

\section{El modelo procesal}

Básicamente, las primeras teorías de la sociología fueron desarrolladas a partir de tres modelos: el mecánico, el orgánico y el procesal. El primero, con sólidas bases en la física -de donde podemos recordar que, para Comte, la sociología era "física social"-incorporó algunos conceptos de dicha disciplina que concluyó en que todo lo social se explicaba con base en interacciones de causas naturales, las cuales eran medibles y explicables conforme a las leyes de la mecánica social.

Utilizando este modelo, se intentaron algunas explicaciones tendientes a comprender el equilibrio y el cambio; sin embargo, los estructuralistas sostenían que todo sistema social tiende a mantener su estado actual hasta que una fuerza ajena, externa a la estructura, alteraba dicho equilibrio promoviendo que diversas fuerzas propias se esforzaran de tal modo que lograban recuperar la estabilidad cuasiestática que le caracterizaba.

Los sistemas sociales, señalaban, se apoyan en códigos, instituciones, tradiciones e intereses; entonces, si un orden social experimenta un cambio se puede conjeturar que hubo una fuerza 
invasora o insurgente que alteró dicho estado anterior. El cambio no es sino la alteración del equilibrio persistente. Los cambios, en este sentido, se consideraban incidentales, ajenos o extrínsecos.

El modelo orgánico en ciencias sociales también atrajo para sí los conceptos y categorías de "sistemas", "subsistemas" y la certeza de que existe una interrelación entre sus partes. Sin embargo, lo que podríamos observar como organismo social poco o nada tiene que ver con un tipo particular de organismo biológico. Walter Buckley argumenta -y le asiste la razón- que la sociedad se asemeja sólo en cuanto a la dependencia mutua entre las partes integrantes del todo, pero eso es también semejante a lo que se sostenía en el modelo mecánico.

El problema de haber equiparado a los sistemas sociales con los organismos vivos fue que los comparaban con el organismo individual, no con la especie en general. Y ello resulta confuso porque el propio darwinismo biológico hace referencia a las especies, no a un organismo, no a los individuos. En todo caso tendría más sentido afirmar que las sociedades son como las especies dado que tanto la conservación como el cambio son propiedades intrínsecas de las especies y de las sociedades: ni las unas ni las otras mueren, como sí lo hace el organismo individual. ${ }^{7}$

Los primeros sociólogos se sirvieron de ambos modelos para lanzar sus teorías sobre la sociedad. Hablemos, por citar ejemplos, de Herbert Spencer y Emile Durkheim. Spencer, por un lado, básicamente argumentaba que las sociedades se comportan de manera similar a un organismo vivo y creía firmemente en la evolución social. Más orientado hacia el individualismo metodológico, construyó teorías sobre los fenómenos sociales que fueron duramente criticadas por su elitismo -especialmente por sus analogías y frases como la "supervivencia del más apto"-; además de ello, a pesar de que sostuvo el carácter evolutivo de las sociedades humanas, no argumentó cómo la historia como disciplina pudiera contribuir a las explicaciones sociológicas, sobre todo, en cuanto a la cooperación que, según él, es el fundamento de la vida social. La cooperación, como fenómeno social que forma parte de nuestra realidad, es histórica puesto que obedece a la cultura, a ciertas coyunturas y contextos que dan forma a la acción del individuo. ${ }^{-}$

Por su parte, Durkheim atrajo a sus teorías la aplicación de ambos modelos de manera más sistemática. Lo anterior puede tener una explicación en que las ciencias naturales ya contaban con un desarrollo aceptable y Durkheim, en su afán por sostener que la sociología es una ciencia, hizo uso de los mismos modelos para explicar fenómenos. Lo anterior puede notarse claramente en sus Reglas del método sociológico cuando clasifica a los fenómenos en normales o patológicos; adicionalmente al uso del modelo orgánico, le concedió un papel a la historia en la medida en que fuera teóricamente útil con fines comparativos para determinar si un hecho social es normal o no: "Después de haber establecido mediante la observación el hecho en general, se remontará a las condiciones que han determinado esta generalidad en el pasado y buscará después si esas condiciones se encuentran todavía en el presente o si, al contrario, han cambiado", ${ }^{2}$ impidiendo que la historia, a manos del sociólogo, superara el papel de antecedente sin convertirse en una poderosa variable explicativa del hecho social -de lo cual deriva la preferencia por la investigación sincrónica por encima de la diacrónica-. 
Heredero de Durkheim, Talcott Parsons también se esforzó por utilizar los modelos orgánico y mecánico en el desarrollo de sus teorías para explicar a la sociedad y es en esa parte donde consideramos que su teoría sobre el sistema social tuvo ciertas carencias dado que, por un lado, consideraba la existencia de los sistemas de creencias que empíricamente son cambiantes e impulsan los procesos morfogenéticos de los sistemas, pero al mismo tiempo dichas creencias, para el autor, podrían considerarse una desviación cuando no concordaban con la estructura establecida, alentando la operación de los mecanismos de control para llevar al sistema social al continuo equilibrio. La historia, disciplina dedicada a dar cuenta -entre otras cosas- de las creencias y cómo éstas dirigen al cambio social, no tenía un lugar claro en su modelo teórico.

Si bien Parsons no descartó la importancia de la historia para el sistema social, la considera ambiguamente dentro de las teorías de la acción que servirían para explicar la realidad social. Parecería que nuestro gran teórico condicionó a la historia a ser parte integrante del sistema siempre y cuando cumpliera con ciertos requisitos de cientificidad o colocándola en la generalidad del sistema y no como producto de las acciones de los individuos:

También hemos reducido nuestra atención a las ciencias que están primariamente organizadas en torno a un esquema teórico distintivo en un nivel analítico. Esto impide claramente la inclusión de la historia como situada en el mismo nivel. En la medida en que la historia sea una ciencia social y no una de las humanidades, no está claramente organizada en torno a ninguno de estos esquemas distintivos, a menos que sea el de los sistemas sociales como un todo. 므

Ambos modelos fueron cayendo en el desuso debido a sus limitaciones. Por un lado, actualmente no podemos argumentar que una sociedad se comporta de manera predeterminada como si se tratara de una máquina; por otro, ambos modelos conferían un papel nulo a la acción de los individuos además de que sostenían que el cambio derivado de algún conflicto era un fenómeno no propio de las estructuras sociales; es decir, las fuerzas de cambio eran externas al sistema social.

Igualmente, es difícil sostener la analogía del organismo vivo semejante a las sociedades dado que "la sociedad, o el sistema sociocultural, no es entonces principalmente un sistema de equilibrio o un sistema homeostático, sino que simplemente nos referiremos a la misma como un sistema adaptativo complejo". 픔

Pero poco después surgió un tercer modelo denominado "procesal", el cual predominó en la Escuela de Chicago en los veinte._- Más cercana a lo empírico y a los actores, se distinguía por hacer uso de la etnografía y del interaccionismo simbólico. El modelo procesal -como lo dice su nombre-descarta que las estructuras sociales sean estáticas:

En esencia, la forma típica del modelo procesal concibe a la sociedad como una interacción compleja, multifacética y fluida, de muy variables grados e intensidades de asociación y disociación. La "estructura" es una construcción abstracta; no es diferenciable del proceso interactivo en desarrollo, sino que 
constituye, más bien, una representación temporaria y acomodaticia de éste en un momento dado. ${ }^{13}$

Este modelo, al observar a la sociedad y sus fenómenos como procesos, tiene la ventaja de guardar mucha más coherencia con el sistema social adaptativo y complejo que describió Buckley debido a la característica "acomodaticia" de las estructuras que se entienden como procesos sociales emergentes; ${ }_{4}^{14}$ éstas pueden presentar cierta forma y función, pero de ninguna manera permanecerán igual durante la existencia de la sociedad de que se trate, ya que dependen tanto del actuar de los individuos como de las circunstancias del entorno. En otras palabras, son adaptativas.

Centrando ahora nuestra atención en el aspecto "interactivo en desarrollo" de un proceso, tenemos que este modelo implica observarlo en el transcurso de su historia; por tanto, si el modelo que está al centro del enfoque sistémico contempla tal desarrollo, no puede ser ahistórico y la adaptación no podrá observarse si no es considerando su pasado y las motivaciones o influencias coyunturales y contextuales que expliquen tal proceso.

Peter Burke define a la historia como "el estudio de las sociedades humanas, destacando las diferencias que hay entre ellas y los cambios que se han producido en cada una a lo largo del tiempo" ${ }_{15}$ la humanización de esta disciplina se traduce en "una nueva preocupación por los hombres y más concretamente por "la gente común" _- por lo que, sostenemos aquí, el acento que la historia pone en los cambios observables en el sistema social, producto de la acción humana, hace que esta disciplina sea imprescindible para el enfoque sistémico. Guillermo Zermeño explica la relevancia que tiene la historia para "comprender el flujo temporal de las relaciones sociales que se tejen, y mediante las cuales se constituye toda existencia y obra humana; de presentes englobados inextricablemente por pasados y futuros" ${ }_{17}$ por lo que si un modelo sistémico pretende explicar algún fenómeno social es imposible apartarse de su historicidad.

De esta manera, el modelo procesal incluye a la historia y el proceso en sí es un elemento que forma parte de cualquier sistema sociocultural. Buckley lo explica de la forma siguiente:

Los sistemas socioculturales son intrínsecamente elaboradores de su estructura y cambiantes; para algunos, los vocablos "proceso" y "cambio" son sinónimos. Dicho en los términos del análisis que realizamos antes, las sociedades y los grupos modifican constantemente sus estructuras adaptándolas a las condiciones internas o externas. Por lo tanto, el proceso concentra su atención en los actos y las interacciones de los elementos integrantes de un sistema en desarrollo, de modo que diversos grados de estructuración de éstos surgen, persisten, se disuelven o cambian. $\frac{18}{}$

La historia tiene un papel fundamental para dar cuenta tanto de la persistencia, de la disolución o del cambio en los sistemas socioculturales. Pero no sólo eso: fenómenos sociales que pueden tener una explicación sistémica (por ejemplo, la delincuencia) pueden observarse en su desarrollo en ciertas etapas históricas de una nación, estado, municipio o comunidad. 
En las ciencias sociales, especialmente en sociología, tradicionalmente utilizamos a la historia para describir los antecedentes de alguna región o fenómeno en particular en lugar de explicar el quehacer humano en el transcurso de ese tiempo; sin embargo, en el diálogo abstracto que se propone entre la TSS en sociología y la historia, esta última disciplina no sólo daría cuenta de un proceso, sino que se elevaría a la categoría de variable explicativa del mismo fenómeno sistémico, tal como veremos en el siguiente apartado.

\section{El modelo de los sistemas axiológicos}

Bertalanffy, al construir su Teoría General de Sistemas, otorga a las variables o condiciones iniciales un papel distinto a la relación causal tradicionalmente utilizada en ciencias sociales. Acuña el término "equifinalidad" que significa, a grandes rasgos, que distintas condiciones iniciales pueden resultar en el mismo estado final.

Posteriormente, Walter Buckley abona el terreno para proponer la "multifinalidad", la cual consiste en que "condiciones iniciales similares pueden llevar a estados finales diferentes"; decir, un sistema puede contar con cierta función de relación de condiciones (o variables) que no necesariamente darán el mismo resultado de un sistema a otro. En este sentido, discutiremos cuál es el papel de la historia como input o condición para la generación de ciertos resultados o estados finales en un sistema.

Sólo para ejemplificar, ¿en qué sistemas puede la historia desempeñar un papel como condición o variable, coadyuvando a que ocurra cierto fenómeno o estado final? No es aventurado sostener que los sistemas sociales se explican por su historia -o, por lo menos, que sin contemplarla la explicación quedaría incompleta- como es el caso de los sistemas político y religioso, el de valores o el económico, que no podrían ser lo que son en un momento en específico si no es como resultado de uno o diversos procesos que les anteceden.

El sociólogo Francisco Parra Luna, en el libro The Performance of Social Systems. Perspectives and Problems, centra su atención en el caso específico de los sistemas axiológicos. Propone un modelo para medir el desempeño de los sistemas sociales donde teóricamente el input o "entrada" está formado por la función entre a) factores estructurales, b) necesidades de los individuos, c) nivel de memoria y d) ambiente. En otras palabras, podemos trasladar este modelo a un sistema de valores existente en cualquier sociedad -por lo menos contemporánea, sin descartar la posibilidad de usarlo en sociedades pasadas-.

Centraremos nuestra atención en dos de esas condicionantes: primero, los factores estructurales, en palabras del propio autor, "son numerosos y complejos [...] [pero] serían: ecológicos, geográficos, geológicos, culturales, religiosos, políticos, demográficos, etc." ${ }^{20} q u e$ dentro de sí mismos contienen procesos históricos singulares y a la vez entretejidos. En cualquier caso, lo anterior significa que un factor estructural como el geográfico puede ser imput condicionante para la generación de un resultado o estado final.

De esta manera, estamos hablando de la ubicación territorial en donde podría incluirse la conformación de una región que, si bien ésta puede tener una alta determinación 
económica,,$\underset{-}{2 \mathrm{I}}$ los procesos sociales que dieron origen a la misma confieren a la historia un papel por desempeñar como condicionante en este conjunto de inputs. Es decir, la ubicación geográfica y la región resultante no son espacios vacíos sino contenedores de una infinidad de procesos históricos, entre otros. Incluso, Sergio Boisier argumenta sobre la existencia de "sistemas territoriales complejos" localizables y definidos por procesos que son necesariamente históricos ${ }_{-}^{22}$ por ello, argumentamos que si vamos a utilizar la ubicación geográfica como factor estructural en un sistema axiológico -como en el caso de Parra Luna- es factible regionalizar atendiendo al desarrollo histórico del lugar logrando así profundizar en la relevancia de dicha variable como condicionante que dará como resultado tal o cual output.

Segundo, el nivel de memoria. Parra Luna lo define como "registro del pasado reciente"; ${ }^{23}$ sin embargo, el autor no señala qué tan reciente debe ser ese pasado; por dicha razón, consideramos que los investigadores tienen libertad de precisar de cuándo a cuándo considerarán su marco temporal para establecer dicho nivel de memoria. Aquí la historia se presenta ofreciendo diversos conceptos clave, entre ellos la memoria histórica, la memoria colectiva o, más reciente todavía, la cultura histórica, ${ }^{24}$ los cuales nos auxilian para definir precisamente qué nivel de memoria vamos a utilizar.

Parra Luna sostiene que

la memoria del sistema representaría, pues, el almacenamiento de datos pasados significativos sobre el mismo, cosa que en los sistemas socioculturales se traduce materialmente en la disposición de bibliotecas con libros de historia, en la sucesión oral de tradiciones, en la acumulación de datos estadísticos o en el mantenimiento de actitudes mentales y formas de vida ancladas más o menos profundamente en los modos de pensar históricos de la colectividad. ${ }^{25}$

Retomando el caso específico del sistema de valores, entendido como un conjunto de ideales que dan pauta a una acción, y que son indudablemente producto histórico humano, ${ }^{26}$ ciertamente éste será válido en una sociedad en un momento determinado. Serían, precisamente, los múltiples cambios en los valores los que podrían ser estudiados por la historia, pero también su permanencia. Es decir, cómo hay valores que se sustituyen por otros o son de plano desechados, pero hay otros que continúan. La propia historia podría responder respecto de las condiciones, actores o sucesos que dieron origen a dichos cambios, desapariciones o continuaciones.

De este modo, podemos elegir estudiar a un sistema de valores sujeto a un contexto y también podríamos analizar los cambios y adaptaciones del mismo sistema en la medida en que está inmerso en un proceso histórico. Utilizando este caso y aplicando el modelo de Parra Luna, tendríamos como hipótesis que la ubicación geográfica de una región o ciudad, sus necesidades económicas, la memoria o cultura histórica -según se decida- y el ambiente serían los inputs de un sistema de valores dado. 
De esta forma, consideramos que por lo menos en el nivel de los sistemas axiológicos, la historia puede estar presente como disciplina fungiendo como antecedente y como contexto, pero también -en ciertos casos y en ciertos niveles del sistema- como condicionante.

\section{La dialéctica}

Para discutir sobre la capacidad o incapacidad de la TSS para explicar los fenómenos dialécticos es necesario comenzar por abordar otra de las críticas hacia este enfoque, la cual no va desligada en forma alguna del supuesto carácter ahistórico: el conservadurismo.

Pablo González Casanova señala que la posición epistemológica de quienes estudian sistemas complejos ha sido conservadora y "cuando deja de serlo, se mantiene en los límites de lo "políticamente correcto" o de lo "académicamente correcto". 27

Parra Luna, desde luego, reconoció en su artículo de los ochenta que existía polémica sobre si la propia TSS era apta para abordar los fenómenos dialécticos; tal crítica se fundamenta en dos aspectos: primero, la relación de la TSS con la cibernética que se traduciría como un intento de mecanización -por tanto, predeterminación- de la conducta humana; segundo, la herencia spenceriana y parsoniana que contiene la TSS y que se relaciona de inmediato con las teorías conservadoras de la sociología.

Ahora bien, epistemológicamente hablando, ¿podemos realmente asumir que existen posturas epistemológicas "conservadoras" cuando la intención de la teoría del conocimiento es indagar la manera en que abordaremos la realidad para explicar un fenómeno social haciendo énfasis en algún aspecto, por ejemplo, el equilibrio? Por ejemplo, si una teoría en ciencias sociales no se centra en la explicación del cambio y el conflicto, ¿es suficiente razón para que, automáticamente, legitime el statu quo? ¿Hasta dónde es posible afirmar que el etiquetar posturas epistemológicas y teóricas de "conservadoras" o "revolucionarias" son adjetivos más bien ideologizados? José Antonio Noguera sostiene que

Urge deshacer el prejuicio esencialista (pero bastante extendido) según el cual adoptar una perspectiva analítica o científica convencional implica necesariamente algo así como "la justificación de lo establecido" o un cierto "conservadurismo" oculto [...] Y es que, como sabía el propio Marx, quien quiera el cambio social hará bien en basarse en una buena teoría científica sobre cómo se produce el mismo y sobre la realidad que se desea cambiar, en vez de caer en una confusión entre punto de vista epistémico e ideología política. $\underline{\underline{28}}$

Vale la pena destacar que el propio Parra Luna argumenta en contra de tal etiqueta conservadora sosteniendo que los fenómenos dialécticos son contemplados y abordados por la TSS desde los trabajos del propio Buckley en los sesenta, además de que la misma puede utilizar el modelo procesal el cual según el mismo autor, "concibe a la sociedad como una interacción compleja, multifacética y fluida, de muy variables grados e intensidades de asociación y 
disociación", ${ }_{2-}$ donde lo único constante es el cambio empatando tal modelo, por tanto, con los fundamentos del pensamiento dialéctico.

Por otro lado, desde los ochenta, Manuel Martín Serrano elaboró un modelo dialéctico para estudiar los sistemas de comunicación del sistema social $3{ }^{\circ}$ que

En el plano teórico y praxeológico intenta dar cuenta de las relaciones que se establecen entre las bases materiales que hacen posible la comunicación (infraestructura), la organización de esas bases materiales reflejo de la organización social que se sirve de ellas (estructura) y el modelo cultural, axiológico e ideológico que se articula con ella (superestructura) [...] [y] trata de identificar las contradicciones que son internas al Sistema Comunicativo y aquellas que se generan en/por relación al Sistema Social. ${ }^{31}$

Parece entonces que el problema no es tanto la supuesta postura "conservadora" de la TSS ni que exista incapacidad para abordar los fenómenos dialécticos desde este enfoque; tal vez la cuestión resida en la confusión que existe entre lo que es una postura ideológica y una epistemológica -como bien señala Noguera-.

Sin duda, el pensamiento crítico está acostumbrado a utilizar las herramientas de la dialéctica para explicar fenómenos sociales, pero ello no significa que tales corrientes teóricas y epistemológicas estén reservadas para unos cuantos. Es responsabilidad de nosotros, como científicos sociales, reconocer la utilidad de una teoría, sea cual sea, dependiendo del problema de investigación al que nos estemos enfrentando.

Tenemos, en resumen, que la TSS es perfectamente capaz de abordar fenómenos dialécticos dado que desde un inicio Buckley afirmó que el cambio y el conflicto forman parte inherente de

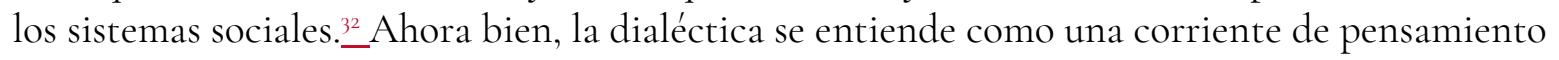
que presta especial interés al desarrollo de los acontecimientos donde el cambio es lo único que permanece; por tanto, la dialéctica es necesariamente histórica. Si la TSS puede utilizar modelos dialécticos, por deducción lógica la TSS puede ser histórica. Como ya vimos anteriormente, la historia se especializa en la identificación de los cambios que la dialéctica señala. Atinadamente, González Casanova coincide con nuestro argumento: además de plantear que el pensamiento crítico posee las herramientas necesarias para construir sistemas alternativos, señala que los sistemas son históricos. El autor señala, sin equivocarse, que los problemas

el pensamiento crítico los plantea a partir de sujetos en formación que buscan construir sistemas universales y complejos alternativos con fuerzas que participan en su construcción. Esa toma de posición no pierde por ello su carácter científico: al contrario, puede advertir, entender y construir la historicidad del sistema y la emergencia de un sistema alternativo [...] En todo caso es a partir del pensamiento crítico como se resuelven más profundamente las contradicciones y la dialéctica de los sistemas complejos en tanto sistemas históricos. 33 
Cuando sostenemos que nos enfrentamos a problemas complejos, no sólo estamos haciendo una invitación a otras disciplinas a aportar perspectivas de análisis -a veces, soluciones- a los mismos problemas. También hacemos hincapié en que dichos problemas pueden bien abordarse sincrónicamente, pero el nivel de complejidad puede hacer la invitación extensiva a investigarlos en su desarrollo a través del tiempo; es decir, a embarcarnos en una investigación diacrónica de los sistemas, sean alternativos o no.

En todo caso, el investigar un sistema "tal y como está" explicando su presente haciendo uso de la dialéctica puede ofrecer las respuestas necesarias para saber por qué "funciona" así y, en caso de ser necesario, proponer la construcción de uno nuevo por parte de los individuos. Recordemos que para la TSS los sujetos no son títeres de la estructura, pero tampoco son totalmente independientes; no obstante, se requiere de la colectivización de las acciones individuales para construir o transformar al sistema sabiendo de antemano qué es lo que se pretende cambiar y las razones para ello. Así surge la pregunta: ¿cómo una acción individual se transforma en colectiva? La respuesta, pensamos, está en los mecanismos sociales.

Los mecanismos sociales

La noción de mecanismo social aparentemente alude a los microfundamentos de los fenómenos de interés para las ciencias sociales, en particular, para la sociología. No obstante, si bien se intenta develar y desentrañar analíticamente una "caja negra" cuyo contenido nos puede revelar qué variables o condiciones influyen para llegar a un resultado de manera individual, consideramos, por otro lado, que este tipo de explicaciones nos remite de igual manera a la conexión que existe entre aquello que hacen los actores (en cierto nivel de análisis) con los resultados colectivos en los cuales centramos nuestra atención.

Felipe González, en su artículo titulado "Los mecanismos sociales y su relación con la distinción micro-macro" publicado en 2016 en la revista Cinta de Moebio, sostiene que si bien de primera mano tales mecanismos se refieren a actores y sus acciones, las diversas definiciones que exponen a los mismos mecanismos como "mediadores" colaboran en nuestra intención de relacionar al mecanismo social con el sistema complejo en el cual aquél se presenta.

Las definiciones propuestas por Leuridan, a diferencia de las de Mahoney, no son excluyentes, pues los mecanismos como mediadores bien pueden ayudar a comprender cómo componentes de órdenes inferiores se concatenan para derivar en fenómenos emergentes (sistemas complejos), o bien, en fenómenos que reflejan agregación de acciones individuales. Y estos mecanismos pueden ser constructos analíticos no observados o bloques de teorías sin contenido empírico. Como iremos viendo, la definición de mecanismos como mediadores es de orden más práctico, se apega más al uso que hacen los cientistas sociales, y permite clarificar en qué medida los mecanismos sociales se constituyen en componentes de sistemas complejos y contribuyen a la generación de teorías. ${ }^{34}$ 
Ahora bien, quizá sea pertinente responder a la siguiente pregunta: ¿la TSS contempla la existencia de los sujetos en su modelo teórico? En primer lugar, no hay un solo modelo teórico en la TSS sino varios, y de muy diversa índole. En segundo, todo depende de qué problema de investigación se trate y de nuestra postura epistemológica, pues, a partir de ahí podremos incluir o no a los individuos dentro del modelo; es decir, depende del investigador. Hemos hablado, por ejemplo, de los modelos utilizados para explicar sistemas de valores. En este último caso, por lo menos, la existencia de los individuos es inobjetable.

De manera general, en su propuesta de modelo para medir el desempeño de un sistema social, Parra Luna señala la necesidad de la existencia de los sujetos en los sistemas complejos de la manera siguiente:

El método y origen central del proceso de teorización consiste, entonces, de entender las necesidades de la población y asumir el punto de vista del grupo como un todo, el cual está integrado por todos y cada uno de los individuos que tienen ese punto de vista. En este enfoque propuesto, los humanos son el eje del sistema, son su razón de ser, por quienes el sistema operará y para quienes los CAMBIOS deben tener un significado positivo. 35

Entonces surge otra pregunta: ¿de qué manera los individuos construyen sistemas? En la medida en que al menos dos sujetos se relacionen entre sí y que una vez producido un cambio en alguno de ellos, éste afecte al conjunto completo, tendremos un sistema. Ahora bien, ¿cómo funciona esa interrelación -bajo qué mecanismos trabaja- de tal forma que ese cambio pueda ser objetivamente observado en los demás miembros del sistema? Cuando hablamos de mecanismos sociales no hacemos referencia al modelo mecánico que se discutió al principio de este artículo. Desde hace mucho tiempo, diversos científicos sociales se han dado a la tarea de construir estos componentes para explicar cómo la acción de una persona lleva a otra a imitarla construyendo, de tal modo, una acción colectiva. Esto quiere decir, sostenemos, que ahí reside la explicación de cómo los individuos generan sistemas: a través de los mecanismos sociales.

Peter Hedström sostiene que "la idea central que subyace al enfoque de los mecanismos es que explicamos un fenómeno social haciendo referencia a una constelación de entidades y actividades, normalmente actores y sus acciones, que están vinculadas entre sí de tal modo que producen regularmente el tipo de fenómeno que queremos explicar". ${ }_{36}^{6}$ Es decir, en el proceso de creación de un sistema consideramos pertinente colocar el acento en aquellos actos que realizan los sujetos y que dan como resultado la existencia de un sistema. Yendo un poco más allá, diferentes científicos sociales se han dado a la tarea de indagar las condicionantes de tales acciones; es decir, qué variables influyen para que una persona actúe de uno o de otro modo.

La preocupación por explicar cómo una acción individual puede colectivizarse fue explícita en James Coleman: abundan las teorías que identifican cuando los fenómenos colectivos (estructurales) tienen influencia sobre otros fenómenos colectivos y más aun sobre los agentes individuales -relaciones tipo I-; también es usual en sociología explicar cómo los agentes individuales pueden determinar la conducta de otros agentes -relaciones tipo 2-. El problema 
viene cuando buscamos analizar cómo los fenómenos individuales se transforman en colectivos -relaciones tipo $3-$.

Sin embargo, son las relaciones del tipo 3 las que han constituido el principal obstáculo, tanto para la investigación empírica como para la teoría que trata las relaciones del nivel macro mediante el individualismo metodológico. Por ejemplo, en su análisis del protestantismo y el capitalismo, Max Weber muestra a través de ejemplos el efecto de la doctrina protestante sobre los valores individuales (relación tipo 2) y, de nuevo a través de ejemplos, el efecto de estos valores sobre las orientaciones de la conducta económica. Lo que no muestra es cómo estas orientaciones individuales se combinaron para producir la estructura de organización económica que llamamos capitalismo (si es que al combinarse produjeron de hecho este efecto). ${ }^{37}$

Dejando clara la importancia de la relación que existe entre los mecanismos sociales y los sistemas complejos, debemos ahora argumentar cómo la historia tiene un lugar específico si buscamos profundizar en las condicionantes que dan como resultado cierta acción del sujeto. En primer lugar, consideramos relevante mencionar que Peter Hedström señala algunos de los mecanismos que podrían ser útiles para saber cómo las acciones individuales pueden transformarse en colectivas y así formar sistemas a través de sus interacciones: el llamado "pensamiento desiderativo" cuyo autor es Donald Davidson, el "síndrome de las uvas amargas" de Jon Elster; el de "formación del deseo impulsado por la disonancia" de Festinger; los mecanismos de "imitación racional" de Hedström, el denominado "profecía que se autocumple" de Robert Merton, entre otros..$^{38}$

Centrando nuestra atención en el mecanismo propuesto por Davidson, tenemos que una persona realiza cierta acción no nada más porque ésta le parece atractiva; para conocer el mecanismo subyacente de un acto debemos explicar qué considera atractivo del acto mismo. Davidson señala que al ser favorables hacia una clase de acción determinada, debemos considerar "actitudes tales como los impulsos, instintos y una gran variedad de convicciones morales, principios estéticos, prejuicios económicos, convencionalismos sociales, metas y valores públicos y privados", 39 que en su conjunto tienen una profunda relación con la historia tanto social como personal del actor; es decir, ¿de qué manera un sujeto tendría convicciones morales si no es como resultado de un proceso histórico tanto colectivo como individual? Por ejemplo, sería válido observar un valor público (como sería la paz o la solidaridad) no sólo como un hecho dado o como un dato más, sino profundizar hasta conocer por qué el mismo valor existe en cierta población atendiendo al bagaje histórico que cualquier valor contiene en sí mismo, quiénes lo reproducen y bajo qué mecanismos lo hacen.

En el caso del mecanismo de imitación racional propuesto por Hedström, el autor lo define de la siguiente manera: "se refiere a una situación donde un actor actúa racionalmente con base en sus creencias las cuales han sido influenciadas por observar las elecciones pasadas de los demás", ${ }^{40}$ donde es importante enfatizar que dichas "elecciones pasadas" responden también a un proceso histórico en el cual el sujeto está irremediablemente inmerso. Tal sería el caso de una 
comunidad continuamente acosada por tribus externas que decide no defenderse frente a cierta situación de inestabilidad social basándose en la memoria histórica y lo que los sujetos han aprendido e internalizado de ésta.

De esta manera, sería absurdo señalar que las intenciones, decisiones y actos de los individuos son ajenos a su contexto, el cual obliga a mirar hacia atrás para comprenderlo y para explicar también qué significado tiene para el actor, de tal manera que lo lleva a elegir entre uno u otro tipo de acción. Mario Bunge aclara, en este sentido, que "todos somos miembros de sistemas sociales y, como tales, actuamos bajo el peso de la sociedad; pero a veces podemos reaccionar para modificar parcialmente la estructura del sistema" ${ }_{-} \mathrm{y}$ es precisamente esa reacción motivada por ciertos elementos- la que abordan los mecanismos sociales.

Si entendemos a la historia como la narración de los grandes acontecimientos políticos del mundo a través del tiempo, obviamente no tendría nada qué hacer en los mecanismos sociales. No obstante, la denominada Escuela de los Anales transformó la forma de trabajar de los historiadores para crear lo que conocemos como "nueva historia" en la que no sólo los grandes personajes adquieren un papel relevante y transformador, sino también los demás individuos que anteriormente pasaban desapercibidos. ${ }^{42}$

Por otro lado, sostenemos que al enfocarse la historia en los agentes, y en lo social, surge necesariamente el aspecto anteriormente abordado que se refiere a la memoria histórica o a la cultura histórica. El actuar inicial de un individuo puede obedecer a lo experimentado en el pasado, a aquellos sucesos que recuerda él y también su comunidad y también puede actuar conforme al recuerdo de lo que dice el texto escrito y oficial. La relevancia de la memoria histórica para explicar las acciones de los agentes dependerá, obviamente, del problema de investigación que estemos abordando.

\section{A manera de conclusión}

Durante la existencia de las ciencias sociales, multiplicidad de teorías y enfoques han surgido y todos, sin excepción, han sido foco de críticas a veces certeras, a veces infundadas debido no a lo malévolo del entorno científico social, sino a la incomprensión de la teoría. Tal como expusimos en este artículo, tenemos diversas maneras de contraargumentar que la TSS sea ahistórica -así como que sea conservadora, como también señalamos-dado que estamos tratando de un enfoque que se ha distinguido por tener una flexibilidad mayor en comparación con las tradiciones estructural funcionalista y voluntarista de las ciencias sociales.

Como sabemos, tanto el modelo orgánico como el mecánico tienen su benevolencia teórica y han inspirado teorías funcionalistas en diversos niveles de análisis y abstracción; sin embargo, aparentemente son acercamientos inacabados que no logran dar cuenta de otro tipo de procesos, como el hecho irrefutable de que las sociedades son adaptativas; es decir, encierran procesos, conflictos y cambios que se dirigen hacia la adaptación del sistema completo que los modelos mencionados anteriormente son incapaces de explicar. De ahí que el modelo procesal arroje luz sobre las insuficiencias de aquellos modelos ya en desuso. 
Por el lado de los sistemas axiológicos, ¿cómo podríamos negar que la existencia misma de los diversos sistemas de valores tiene una explicación histórica? El papel que esta disciplina desempeña ya sobrepasó el clásico "antecedente del fenómeno" para convertirse en una condicionante para la existencia de éste; es decir, su ubicación teórica es distinta y más relevante de lo que pensábamos. Como parte de un conjunto de inputs, entre los cuales se encuentran diversos factores estructurales, consideramos que el propio territorio y su regionalización obedecen a diversos factores históricos, tal como han discutido numerosos regionalistas, historiadores y antropólogos; pero en el momento en que el modelo de sistemas axiológicos coloca el acento también en el nivel de memoria como condicionante para la existencia de cierto sistema de valores, nos lleva a revisar qué recuerda un conjunto de actores acerca del pasado que podría explicar por qué un valor impera en su comunidad o los motivos de su desaparición, según sea el caso.

Del mismo modo, consideramos que el propio pensamiento dialéctico tiene muchos elementos que aportar a los sistemas complejos sin estancarse en las contradicciones, sino avanzando hacia una descripción e interpretación mucho más completa de los fenómenos sociales que hoy en día urge resolver. La dialéctica es necesariamente histórica; por tanto, abriendo los canales de comunicación entre ésta y la TSS, no hay razón alguna para sostener que estaríamos construyendo modelos ahistóricos. Si bien Pablo González Casanova argumenta que la TSS ha sido utilizada de manera conservadora al aplicarse más a las técnicas y otras disciplinas que sostienen al sistema capitalista, el autor abre la discusión para que haya una comunicación y aprovechamiento teórico, conceptual y político entre el pensamiento crítico y el sistémico.

Por otra parte, sostenemos que, si bien los diversos modelos de mecanismos sociales no fueron elaborados explícitamente para utilizarse en convergencia con la TSS, también podemos sostener que la flexibilidad y sólido fundamento de los mismos modelos los convierten en una herramienta ideal si pretendemos explicar cómo la acción de un sujeto puede colectivizarse y formar sistemas complejos. De la mano iría el papel de la memoria histórica y colectiva -o cultura histórica- que influye en los sujetos en el sentido de la posibilidad de que el pasado sea una de las razones por las cuales un individuo hace lo que hace.

Es probable que, como escribió Parra Luna, la TSS sea el enfoque sociológico por excelencia debido a que la propia bondad de los modelos que pueden construirse dejan la puerta abierta a aproximaciones que en un primer momento pensaríamos incompatibles, como el funcionalismo. Si bien los fenómenos sociales, bajo el pensamiento sistémico, se tornan complejos, consideramos que ahí reside la oportunidad de aprovechar la rica variedad teórica y metodológica que distingue a las ciencias sociales y humanas, así como el desafío para los investigadores que apostamos por modelos que nos acerquen a una explicación y comprensión más integral y completa de los problemas de investigación, sobre todo los más apremiante

\section{Referencias}

AGÜERO, Juan Omar. "Niklas Luhmann y los sistemas autopoiéticos". VI Jornadas de Sociología de la UNLP. La Plata: Universidad Nacional de La Plata, Facultad de Humanidades y Ciencias 
de la Educación, Departamento de Sociología, 20Io, I-I4. https://www.aacademica.org/ooo027/38.pdf

AZPÚRUA GRUBER, Jesús. "La Escuela de Chicago. Sus aportes para la investigación en ciencias sociales". Revista Universitaria de Investigación(2) (julio-diciembre 2005): 2535. http://www.redalyc.org/pdf/410/4IO21705003.pdf.

BOISIER, Sergio. "Una (re)visión heterodoxa del desarrollo (territorial): un imperativo categórico". Territorios (II) (2004). https://www.redalyc.org/articulo.oa?id=3570IIO4

BUCKLEY, Walter. "Society as a Complex Adaptative System". En Modern Systems Research for the Behavioral Scientist, ed. Walter Buckley, 490. Chicago: Aldine Publishing Company, I968.

--_-_. La sociología y la teoría moderna de los sistemas. Buenos Aires: Amorrortu Editores, 1982.

BUNGE, Mario. Ser, saber y hacer. México: Paidós, Universidad Nacional Autónoma de México, 2002.

BURKE, Peter. Sociología e historia. Madrid: Alianza Editorial, ı980.

Formas de hacer historia. Madrid: Alianza Editorial, I99I.

CARRASQUEL JEREZ, Carmen H. "El fin de la historia. Reflexiones sobre lo inacabado del conocimiento histórico". Procesos Históricos (30) (2016): 96-IOI.

COLEMAN, James. "Teoría social, investigación social y teoría de la acción”. En Teoría sociológica analítica, ed. José Antonio Noguera, II5-I44. Madrid: Centro de Investigaciones Sociológicas, 2OIO.

DAVIDSON, Donald. Ensayos sobre acciones y sucesos. México: Crítica, Universidad Nacional Autónoma de México, 1995.

DURKHEIM, Emile. Las reglas del método sociológico. México: Fondo de Cultura Económica, I997.

GARCÍA SÁNCHEZ, Ángel. “Apuntes para una crítica ilustrada a Niklas Luhmann”. Endoxa: Series Filosóficas (II) 27I295. http://revistas.uned.es/index.php/endoxa/article/download/4935/4755

GONZÁlEZ CASANOVA, Pablo. Las nuevas ciencias y las humanidades. De la academia a la política. Barcelona: Anthropos Editorial, 2005.

GONZÁLEZ, Felipe. "Los mecanismos sociales y su relación con la distinción micromacro". Cinta de Moebio: Revista de Epistemología de las Ciencias Sociales (55) (2016): I628. http://www.moebio.uchile.cl/55/gonzalez.html 
HEDSTRÖM, Peter. "Rational Imitation". En Social Mechanisms. An Analytical Approach to Social Theory, ed. Peter Hedström y Richard Swedberg, 306-327. Nueva York: Cambridge University Press, 2005.

"La explicación del cambio social: un enfoque analítico". En Teoría sociológica analítica, ed. José Antonio Noguera, 2II-235. Madrid: Centro de Investigaciones Sociológicas, 20 o.

MARTÍN SERRANO, Manuel. "El modelo dialéctico de la comunicación". En Teoría de la comunicación I. Epistemología y análisis de la referencia, Manuel Martín Serrano et al. 159-74. Madrid: Alberto Corazón, I98I. http://eprints.ucm.es/izir6/

NOGUERA, José Antonio. "Por qué necesitamos una sociología analítica". En Teoría sociológica analítica, ed. José Antonio Noguera, I7-38. Madrid: Centro de Investigaciones Sociológicas, 2OIO.

NÚÑEZ RODRÍGUEZ, Carlos Juan. "Para una crítica a la teoría de sistemas de Luhmann. El EZLN como movimiento de protesta”, Gestión y Estrategia (47) (enero-junio 2015): 2336. http://zaloamati.azc.uam.mx/handle/ingi/2939

PARRA LUNA, Francisco. “¿Para qué sirve la teoría de sistemas en sociología?”. Revista Española de Investigaciones Sociológicas (I5) (I98I): 77-III.

. "Towards an Axiological System Theory" En The Performance of Social Systems. Perspectives and Problems, ed. Francisco Parra Luna, 37-57. Madrid: Springer Science+Business Media, Universidad de Madrid, 2000.

. "A Model for Measuring The performance of Social Systems" En The Performance of Social Systems. Perspectives and Problems, ed. Francisco Parra Luna, 89-II6. Madrid: Springer Science+Business Media, Universidad de Madrid, 2000 .

"Hacia la localización del 'lugar de la Mancha' en el Quijote: un enfoque sociosistémico como esbozo metodológico". Cuadernos de Estudios Manchegos (28) (2004): 219-238.

"El 'pecado social' de la sociología. Una reflexión crítica desde la axiología sistémica". Anduli: Revista Andaluza de Ciencias Sociales (7) (2007): 23-44.

Elementos para una teoría formal del sistema social. Madrid: Editorial Complutense, 2009, 268.

. ¿¿Por qué Villanueva de los Infantes es el 'lugar de La Mancha’ en El Quijote?”. Barataria: Revista Castellano-Manchega de Ciencias Sociales (I8) (2014): I8I-I93.

PARSONS, Talcott. El Sistema Social. Madrid: Alianza, i988. 
RODRÍGUEZ SÁNCHEZ, Ángel. "La Nueva Historia. Una introducción”. Norba. Revista de Historia (5) (I984): 205-2I2.

SÁNCHEZ COSTA, Fernando. "La cultura histórica. Una aproximación diferente a la memoria colectiva". Pasado y Memoria. Revista de Historia Contemporánea (8) (2009): 267286. http://www.culturahistorica.es/sanchez_costa/cultura_historica.pdf.

SÁNCHEZ FLORES, Mónica Judith. "La filosofía del sujeto y la sociología del conocimiento en las teorías de Jürgen Habermas y Niklas Luhmann". Confines de Relaciones Internacionales y Ciencia Política (5) (2007): 8798. http://www.scielo.org.mx/scielo.php?script=sci_arttext\&pid=SI870$35692007000100008 \& \operatorname{lng}=\mathrm{es} \& n \mathrm{~nm}=\mathrm{is0}$

SCHWANDT, David y David SZABLA. "Structuration Theories and Complex Adaptive Social Systems: Inroads to Describing Human Interaction Dynamics". En Emergence: Complexity and Organization (2013). DOI: Io.emerg/I0.17357.83ao2f74fdzac7032bic947f9fao5ood

SESENTO GARCÍA, Leticia. Modelo sistémico basado en competencias para instituciones educativas públicas. Tesis de Doctorado, Centro de Investigación y Desarrollo del Estado de Michoacán, 2008.

URTEAGA, Eguzki. "La teoría de sistemas de Niklas Luhmann". Contrastes. Revista Internacional de Filosofía, vol. XV (2ого): 3ог-3i7.

VAN YOUNG, Eric. "Haciendo historia regional: consideraciones metodológicas y teóricas". En Región e historia en México (1700-1850). Métodos de análisis regional, comp. Pedro Pérez Herrero, 99-I22. México: Instituto Dr. Mora, Universidad Autónoma Metropolitana, I99i.

WAN, Poe Yu-Ze. "Dialectics, Complexity and the Systemic Approach: Toward a Critical Reconciliation". Philosophy of the Social Sciencies (4) (2012). https://doi.org/IO.II77/0048393II244I974

ZERMEÑO, Guillermo. "Historia y Grafía, siete años después". Historia Mexicana 50(4) (20oo): 945-7I. https://historiamexicana.colmex.mx/index.php/RHM/article/view/I35I/I2I4

Notas

I Francisco Parra Luna, “¿Para qué sirve la teoría de sistemas en sociología?”, Revista Española de Investigaciones $\quad$ Sociológicas (I5) $\quad$ (I98I): II4, https://dialnet.unirioja.es/servlet/articulo?codigo $=273567$

2 Véase Ángel García Sánchez, "Apuntes para una crítica ilustrada a Niklas Luhmann", Endoxa: Series Filosóficas (II) (I999); Carlos Juan Núñez Rodríguez, "Para una crítica a la teoría de sistemas de Luhmann. El EZLN como movimiento de protesta", Gestión y Estrategia (47) (enero-junio 2015). 
3 Véase Mónica Judith Sánchez Flores, "La filosofía del sujeto y la sociología del conocimiento en las teorías de Jürgen Habermas y Niklas Luhmann", Confines de Relaciones Internacionales y Ciencia Política (5) (2007); Eguzki Urteaga, "La teoría de sistemas de Niklas Luhmann", Contrastes. Revista Internacional de Filosofía, vol. XV (2ого).

4 David Szabla y David Schwandt, "Structuration Theories and Complex Adaptive Social Systems: Inroads to Describing Human Interaction Dynamics", Emergence: Complexity and Organization (2013). DOI: Io.emerg/I0.17357.83ao2f74fd3ac7032bic947f9fao5ood

5 Otro problema del enfoque sistémico de Luhmann consiste en que cada crítica la responde construyendo conceptos nuevos con el mismo nivel de abstracción o aun más elevado, lo cual complica comunicar su teoría general con la historia. Véase Juan Omar Agúero, "Niklas Luhmann y los sistemas autopoiéticos", VI Jornadas de Sociología de la UNLP (La Plata: Universidad Nacional de La Plata. Facultad de Humanidades y Ciencias de la Educación. Departamento de Sociología, 2oio).

6 Francisco Parra Luna, The Performance of Social Systems. Perspectives and Problems (Madrid: Springer Science+Business Media, Universidad de Madrid, 20oo); "Hacia la localización del 'lugar de la Mancha' en el Quijote: un enfoque sociosistémico como esbozo metodológico", Cuadernos de Estudios Manchegos (28) (2004); "El 'pecado social' de la sociología. Una reflexión crítica desde la axiología sistémica", Anduli: Revista Andaluza de Ciencias Sociales (7) (2007); “¿Por qué Villanueva de los Infantes es el 'lugar de La Mancha' en El Quijote?", Barataria: Revista Castellano-Manchega de Ciencias Sociales (I8) (2014).

7 Durkheim comprendió esta diferencia y así lo argumenta en Las reglas del método sociológico, pero ello no significó que abandonara al modelo orgánico. Walter Buckley hace un recuento más amplio sobre los principales conceptos, analogías y problemas de los modelos mecánico y orgánico en el libro La sociología y la teoría moderna de los sistemas (Buenos Aires: Amorrortu Editores, 1982).

8 Carmen H. Carrasquel Jerez, "El fin de la historia. Reflexiones sobre lo inacabado del conocimiento histórico", Procesos Históricos. Revista de Historia y Ciencias Sociales (zo) (juliodiciembre 2016).

9 Emilio Durkheim, Las reglas del método sociológico (México: Fondo de Cultura Económica, 1997), 108.

Io Talcott Parsons, El sistema social (Madrid: Alianza, I988), 352. https://teoriasuno.files.wordpress.com/2013/o8/el-sistema-social-talcott-parsons.pdf

II Walter Buckley, "Society as a Complex Adaptative System", en Modern Systems Research for the Behavioral Scientist (Chicago: Aldine Publishing Company, I968), 490. Traducción propia.

I2 Un excelente recuento de la influencia y posturas de la Escuela de Chicago puede encontrarse en Fernando Jesús Azpúrua Gruber, "La Escuela de Chicago. Sus aportes para la 
investigación en ciencias sociales", Revista Universitaria de Investigación (2) (julio-diciembre 2005): 25-35, http://www.redalyc.org/pdf/410/41021705003.pdf

I3 Walter Buckley, La sociología y la teoría moderna de los sistemas (Buenos Aires: Amorrortu Editores, I982), 36-37.

I4 Leticia Sesento García, "Modelo sistémico basado en competencias para instituciones educativas públicas" (Tesis de doctorado, Centro de Investigación y Desarrollo del Estado de Michoacán, 2008).

I5 Peter Burke, Sociología e historia (Madrid: Alianza Editorial, I980), II.

I6 Ángel Rodríguez Sánchez, "La Nueva Historia. Una introducción”, Norba Revista de Historia (5) (1984): $2 \mathrm{IO}$.

I7 Guillermo Zermeño, "Historia y Grafía, siete años después", Historia Mexicana 50(4) (200) (abril-junio 200I):

http://historiamexicana.colmex.mx/index.php/RHM/article/view/I35I/I2I4

947 ,

I8 Buckley, La sociología, 37 .

19 Ibid., 98

20 Francisco Parra Luna, "A Model for Measuring the Performance of Social Systems", en The Performance of Social Systems. Perspectives and Problems, ed. Francisco Parra Luna, 9I (Nueva York: Springer Science + Business Media, 2000). Traducción propia.

2I Cfr. Eric Van Young, "Haciendo historia regional: consideraciones metodológicas y teóricas", en Región e historia en México (1700-1850). Métodos de análisis regional, comp. Pedro Pérez Herrero (México: Instituto Dr. Mora, Universidad Autónoma Metropolitana, I99I).

22 Cfr. Sergio Boisier, "Una (re)visión heterodoxa del desarrollo (territorial): un imperativo categórico", Territorios (II) (2004). https://www.redalyc.org/articulo.oa?id=3570IIO4

23 Parra, "A Model for Measuring", 9o. Traducción propia.

24 Fernando Sánchez Costa, "La cultura histórica. Una aproximación diferente a la memoria colectiva", Pasado y Memoria. Revista de Historia Contemporánea (8) (2009): 267286, http://www.culturahistorica.es/sanchez_costa/cultura_historica.pdf.

25 Francisco Parra Luna, Elementos para una teoría formal del sistema social (Madrid: Editorial Complutense, 2009), 268. 
26 Carmen H. Carrasquel Jerez, "El fin de la historia. Reflexiones sobre lo inacabado del conocimiento histórico", Procesos Históricos. Revista de Historia y Ciencias Sociales (zo) (juliodiciembre 2016).

27 Pablo González Casanova, Las nuevas ciencias y humanidades. De la academia a la política (Barcelona: Anthropos Editorial, 2005), 209.

28 José Antonio Noguera, "Por qué necesitamos una sociología analítica", en Teoría sociológica analítica, ed. José Antonio Noguera, 32-33 (Madrid: Centro de Investigaciones Sociológicas, 2010).

29 Buckley, La sociología, 36.

30 Manuel Martín Serrano, "El modelo dialéctico de la comunicación", en Teoría de la Comunicación I. Epistemología y análisis de la referencia, Manuel Martín Serrano et al., I59-I74 (Madrid: Alberto Corazón, I98I) http://eprints.ucm.es/IziI6/

3I Ibid., 5.

32 Poe Yu-ze Wan realiza una interesante discusión sobre la relación que existe entre la dialéctica y el pensamiento sistémico en su artículo "Dialectics, Complexity and the Systemic Approach: Toward a Critical Reconciliation", en Philosophy of the Social Sciencies (4) (2012) https://doi.org/I0.II77/004839311244I974

33 González, Las nuevas ciencias, 2I2-2I4.

34 Felipe González, "Los mecanismos sociales y su relación con la distinción micromacro", Cinta Moebio: Revista de Epistemología de las Ciencias Sociales (55) (2016), https://www.moebio.uchile.cl/55/gonzalez.html

35 Parra, "A Model for Measuring", ro2. Resaltado en el original. Traducción propia.

36 Peter Hedström citado en José Antonio Noguera, "Por qué necesitamos una sociología analítica", 3г.

37 James Coleman, "Teoría social, investigación social y teoría de la acción", en Teoría sociológica analítica (Madrid: Centro de Investigaciones Sociológicas, 2010), I29-I30.

38 Peter Hedström, "La explicación del cambio social: un enfoque analítico", en Teoría sociológica analítica (Madrid: Centro de Investigaciones Sociológicas, 20го), 216.

39 Donald Davidson, Ensayos sobre acciones y sucesos (México: Crítica, Universidad Nacional Autónoma de México, 1995), i8. 
4o Peter Hedström, "Rational Imitation", en Social Mechanisms. An Analytical Approach to Social Theory, ed. Peter Hedström y Richard Swedberg, 307 (Nueva York: Cambridge University Press, 2005). Traducción propia.

4I Mario Bunge, Ser, saber y hacer (México: Paidós, Universidad Nacional Autónoma de México, 2002), II4.

42 Peter Burke, Formas de hacer historia (Madrid: Alianza Universidad, I99I), I5-I6.

\section{Mónica Eugenia Moreno Rubio}

Socióloga y maestra en Ciencias Sociales por la Universidad Autónoma de Querétaro. Actualmente cursa el Doctorado en Estudios Interdisciplinarios sobre Pensamiento, Cultura y Sociedad en la Facultad de Filosofía de la misma universidad, institución donde ha desarrollado actividades de docencia e investigación. Sus principales temas giran en torno a la teoría de sistemas sociales, la sociología analítica y los estudios socioculturales. Ha realizado investigaciones sobre temas como tolerancia, migraciones, elites intelectuales y sistemas de valores. Uno de sus trabajos más recientes es "Teoría de sistemas y mecanismos sociales: los actores actuantes en el proceso de realimentación sistémica", publicado en la Revista RELACSO de la Facultad Latinoamericana de Ciencias Sociales. 